\title{
Formación holística para mejorar el desarrollo de habilidades blandas en estudiantes de la unidad educativa Fiscal Vicente Rocafuerte, Guayaquil, 2020
}

\author{
PHD. Tobar Bohórquez María Leonor \\ maríatobarbohorquez34@gmail.com \\ Unidad Educativa Fiscal Vicente Rocafuerte \\ UEFVR-Guayaquil-Ecuador
}

El holismo se fundamenta en la ciencia, donde se evidencia una compleja red de interrelaciones en las distintas partes de un mundo globalizado, vinculando el aspecto humanístico con la formación educativa de un creciente universo estudiantil respetuoso de los principios, valores y buenas costumbres para un mejor mañana.

\section{RESUMEN}

La presente investigación tiene como objetivo general, establecer un programa de formación holística para mejorar el desarrollo de las habilidades blandas en los estudiantes de nivel bachillerato. Se fundamenta en la teoría de Jan Smuts (1926), sobre "holismo" y de David Goleman (2001), sobre las "habilidades blandas" del individuo, sustentadas con sus respectivas dimensiones e indicadores direccionados para la enseñanza-aprendizaje en la formación de los estudiantes de la unidad educativa fiscal Vicente Rocafuerte. El diseño de la investigación tuvo un enfoque mix to y estudio básico; el tipo de investigación es de campo. La muestra poblacional seleccionada es probabilística y corresponde a 154 estudiantes y 12 docentes del tercer año de bachillerato, la técnica aplicada fue la encuesta la misma que fue validad mediante Alfa de Cronbach 0,826909379, la prueba de normalidad de las variables por KolmogórovSmirnov resultó 0,000 y la correlación de Rho Spearman fue positiva cuyo coeficiente fue mayor a 0 , descartando así, la hipótesis nula Ho: "la formación holística no tiene una relación significativa con las habilidades blandas". La propuesta es, un programa de estudio que permite mejorar las habilidades blandas mediante la formación holística, con ello, optimizar el rendimiento académico de la población estudiantil objeto de investigación.

Palabras Claves: formación holística; habilidades blandas; competitividad. 


\title{
Holistic training to improve the development of soft skills in students of the educational unit attorney Vicente Rocafuerte, Guayaquil, 2020
}

\begin{abstract}
ABSTRAC
The general objective of this research is to establish a holistic training program to improve the development of soft skills in high school students. It is based on the theory of Jan Smuts (1926), on "holism" and David Goleman (2001), on the "soft skills" of the individual, supported by their respective dimensions and indicators aimed at teachinglearning in the formation of the students of the Vicente Rocafuerte fiscal educational unit. The research design had a mixed focus and basic study; the type of research is in the field. The selected population sample is probabilistic and corresponds to 154 students and 12 teachers of the third year of high school, the applied technique was the same survey that was valid using Cronbach's Alpha 0.826909379, the normality test of the variables by Kolmogórov-Smirnov It was 0.000 and the Rho Spearman correlation was positive whose coefficient was greater than 0 , thus ruling out the null hypothesis Ho: "holistic training does not have a significant relationship with soft skills". The proposal is a study program that allows improving soft skills through holistic training and thereby optimizing the academic performance of the student population under investigation
\end{abstract}

Keywords: Holistic Training; Soft Skills; Competitiveness

Artículo recibido: 02 enero 2022 Aceptado para publicación: 28 enero 2022 Correspondencia: maríatobarbohorquez34@gmail.com Conflictos de Interés: Ninguna que declarar 


\section{INTRODUCCIÓN}

Establecer un programa de formación holística para mejorar el desarrollo de las habilidades blandas en los estudiantes de nivel bachillerato, evidencia la importancia y relevancia que en la actualidad se ha dado a las competencias no cognitivas vinculadas a los aspectos intra e interpersonales, así, el aspecto de competitividad ha evolucionado significativamente en relación al desempeño laboral entre el personal que posee perfiles de carácter transversal quienes ponen en relevancia sus valores, su capacidad de renovación y transformación, y; aquel personal con conocimientos especializados que pueden ser adquiridos en la misma organización.

Los centros educativos, constituyen a través de sus niveles de formación un medio fundamental para desarrollar una educación integral para su población estudiantil, sustentada en principios de responsabilidad social, valores y ética como complemento de aquellos contenidos que conforman la malla curricular de sus aprendizajes, elevando así, su perfil académico, profesional y competitivo, que se verán reflejados en lo humanístico y en lo emotivo. A nivel globalizado, es evidente la transformación e impacto que ha tenido el ámbito educativo por efecto del uso de la tecnología que ha generado brechas en la docencia tales como la necesidad del desarrollo del pensamiento crítico, el aprendizaje continuo a través de la convivencia diaria, integración de perspectivas sostenible al currículum, gestión y aplicación práctica de los conocimientos y la virtualización como sistema para la enseñanza y aprendizaje.

Por tal razón, se requiere de las competencias blandas para lograr estrechar las brechas existentes, donde los atributos personales inciden positivamente en la interacción y rendimiento como complemento de las competencias técnicas o disciplinares a desarrollarse en las actividades educativas. Los actuales indicadores del aprendizaje determinan que el individuo debe poseer destrezas orientadas a un constante aprender, desaprender y reaprender, a cambio de dejar de ser actores pasivos para la comunicación y el conocimiento. El objetivo de esta investigación, se orientó en proponer un programa de formación holística para mejorar las habilidades blandas de los estudiantes del tercer año de bachillerato de la unidad educativa fiscal Vicente Rocafuerte de Guayaquil, para lo cual fue necesario diagnosticar las habilidades blandas inmersas en la interacción de los estudiantes, diseñar un programa de formación holística a través de un estudio de campo en el centro de estudio antes mencionado, así como, validar el programa de 
formación holística propuesto. Por lo tanto, cabe el siguiente planteamiento de la problemática:

¿De qué manera incide un programa de Formación Holística para mejorar el desarrollo de las Habilidades Blandas de los estudiantes del bachillerato de la UEF "Vicente Rocafuerte" Guayaquil, 2020?

Los sustentos teóricos desarrollados, están vinculados con las variables de la temática de estudio, donde fueron considerados los antecedentes de investigaciones ya realizadas y de teorías pertinentes. Así se tiene que, para Gluyas et al. (2015), la conexión entre mente y cuerpo genera una dimensión personal en cada individuo, a través de la cual, se desarrolla su formación cognitiva, espiritual y física que fortalece su potencial. Por lo tanto, se aprende a ser, reaprendiendo mediante el autoconocimiento, autogestión y reflexión. Llamazares, (2017), deduce que, los fundamentos epistemológicos del holismo proponen arribar hacia los niveles intuitivos estimulando los procesos cognitivos divergentes y creativos, constituyendo así, una apertura de la lógica no lineal y paradójica. Esto está orientado en resaltar la necesidad que ha tenido el holismo en sustentarse bajo parámetros que lo relacionen con el principio de interdependencia como sujeto que observa o que es observado, para trascender además en conceptos antagónicos tales como materia - espíritu, cualidad - cantidad, duro - blando que buscan concientizar la práctica de actitudes integrales y participativas.

Para López, (2018), el ser humano, manifiesta integridad a base de la comprensión previa de sus actitudes holistas, aportando sus capacidades y tiempo con fines de lograr una formación contínua y trascendente, donde tales cualidades holísticas son procedentes aplicarlas en la actual realidad educativa siglo xxi, por personal docente capacitado para ello, en virtud que, la pérdida de principios y valores se acentúan cada vez más en el comportamiento juvenil, que se verá reflejado en su futura práctica profesional. Esto se evidencia en las conflictivas situaciones sociales por las que atraviesa las colectividades, educativas, productivas, comerciales, políticas, económicas, de salud, religiosas y medio ambientales entre otras, donde prevalecen los intereses materiales sobre los humanitarios. Marrero (2018), afirmó, no es dominio de las habilidades socio emocionales el saber utilizar la tecnología y sus distintas aplicaciones de comunicación que permiten interactuar en tiempo real con los participantes conectados. Por tal razón, en la actualidad cada nivel formativo debe tener como premisa el desarrollar eficientemente la inteligencia 
emocional. Para Tolentino (2020), Los actuales lineamientos educativos, presentan falencias y vacíos en relación a la formación de lideres, donde la educación integral debe ser iniciada desde los primeros años de los procesos educativos, en virtud que una buena educación constituye un instrumento importante para solventar eventos conflictivos mediante formación emocional.

Smuts (1926), desarrolló la teoría sobre holismo y evolución, que pregonaba "el todo no es la mera suma de sus partes, ya que el todo y sus partes se influyen y determinan mutuamente". Según esta teoría, el holismo se conceptúa de modo cognitivo, interpretando y definiendo las partes partiendo del todo, lo que contradice al pensamiento individualista que se direcciona a explicar el todo a partir de sus partes. Esta teoría está sustentada en las inteligencias múltiples de Gardner, las cuales dan soporte al modelo de educación holística, cuyas dimensiones son, dimensión personal, interpersonal y ecológica.

Goleman (2001), fundamentó su teoría sobre habilidades blandas en dimensiones tales como las habilidades sociales, empatía, auto conciencia, auto regulación y motivación, las cuales generan la capacidad de reconocer los propios sentimientos y de los semejantes, la propia motivación, el manejo de las interrelaciones, así como, la persuasión e influencia sobre otras personas, inspiración de liderazgo, manejo de conflictos, trabajo en equipo, colaboración y el desempeño laboral.

Los actuales modelos educativos están poniendo atención a lograr la diversidad para la enseñanza, rescatando valores como la ética, dimensionando el significado de colaboración, participación, tolerancia y empatía enfocadas en el bienestar compartido, donde las herramientas tecnológicas utilizadas como instrumento didáctico facilitan el desarrollo de las habilidades duras y blandas en el estudiante que pasa a tener un rol de autoformación con un docente orientador de los procesos de formación.

Así, para lograr tales aspiraciones, se requiere involucrar aspectos como, la flexibilidad donde no caben los modelos educativos rígidos, en su lugar se debe optar por la adaptabilidad a cada circunstancia que se genere producto de la evolución social y la tecnología, apoyo humanitario, cuidado medio ambiental, etc., en busca de alcanzar modelos educativo sostenibles.

La implementación de una educación holística desde los niveles de formación básica, posibilita fomentar la afectividad, la reflexión, la gestión de emociones, cooperación, 
respeto, autoaceptación, tolerancia, espíritu crítico, creatividad, liderazgo, prevención de conflictos, honradez, etc., evitando así, consolidar una metodología lógico racional solo de habilidades cognoscitivas académicas. Moravec, Cobo, \& Francesc, (2020), consideran que, la sociedad se vuelve mas exigente demandando de los habitantes manifestaciones de multiples cualidades como producto de su formación académica, que evidencien autonomía para el emprendimiento, dominio de las tecnologías, innovación con pensamiento crítico, reflexividad en la solucion de conflictos entre otros, aspectos, los cuales deben ser desarrollados en los procesos de instrucción docentes direccionado a "desaprender para aprender a aprender."

El vínculo entre profesores y alumnos en las sesiones educativas, contienen aspectos físicos, cognitivos y socioemocionales, cuyo dominio son necesarios para un buen desempeño académico, social y laboral. En la actualidad, el perfil estudiantil se direcciona a satisfacer las demandas del mercado laboral, donde se exige eficiencia a más del aspecto de conocimientos, así como, una buena ejecución de la inteligencia emocional. Por tal razón, la actual situación mundial generada por la pandemia, ha acelerado el desarrollo de actividades educativas con modalidad virtual, donde tanto docentes y estudiantes se han visto en la autoexigencia de dominar las actividades tecnológicas propias de la presente situación, las cuales han tenido el objetivo de evitar los contagios, y mantener la continuidad de los procesos de formación en todos los niveles, poniendo de manifiesto las destrezas y habilidades relacionadas con la inteligencia emocional, el trabajo cooperativo, responsabilidad, tolerancia, etc. Por lo tanto, la presencia de este fenómeno, ha tenido un sorprendente impacto entre la tecnología y el ser humano en la diaria convivencia del hogar, ya que docentes y estudiantes realizan la enseñanza - aprendizaje dentro del hogar, que ha originado una diversidad de aspectos emocionales que antes no ocurrían.

Las habilidades blandas se vinculan a la inteligencia emocional que se manifiesta con la capacidad de desarrollar una relación y comunicación de manera afectiva y considerada con otras las personas, son competencias emocionales propias, importantes en el aspecto de productividad empresarial para desarrollar liderazgo en las actividades de comercialización. En relación al ámbito educativo, lo deben poseer los docentes y aprenderlas los estudiantes durante su formación. Así, en los centros de formación siglo xxi, se han identificado diversidad de aspectos para la aplicación de habilidades blandas 
para el desarrollo de la inteligencia emocional en la población estudiantil en proceso de formación, Estas son:

Autoliderazgo, que fortalece la capacidad de dirigir la propia vida. Automotivación, fuente de energía que impulsa a lograr objetivos. Creatividad, capacidad para imaginar, innovar y crear. Comunicación y escucha, don o habilidad para intercambiar información con otras personas. Visión, relacionada a la proyección del alcance de una planificación para logar objetivos. Gestión del tiempo, planificación y organización de tareas y actividades pertenecientes a un proceso. Principios y Valores, que vincula la integridad, responsabilidad y compromiso para llevar a cabo trabajos o tareas.

De igual manera, se tiene la Autoconfianza, fortalezas y debilidades de las capacidades. Resiliencia, adaptación para tratar los propios conflictos. La sociabilidad, amistad, sensibilidad y vínculo emocional. Empatía, Pro actividad, Pensamiento crítico, valoración de los aspectos materiales y espirituales positivos y negativos. Sinergia, colaboración para el trabajo en equipo. Pertenencia a la interculturalidad, aceptación de la diversidad de culturas y sociedades que forman parte de un territorio.

\section{MATERIALES Y MÉTODOS}

El diseño de la investigación tuvo un enfoque mixto, abordados desde aspectos cognitivos emocionales, sociales, artísticos y espirituales, en virtud de considerarlo como el nuevo paradigma educativo, que propone una visión creativa, integral y multidisciplinaria direccionados al desarrollo de pensamientos versátiles vinculados, con el conocimiento, sentimientos y emociones como parte integral del universo del entorno en el que se realizan todas las actividades diarias del ser humano. Es básica, ya que está dirigida a un conocimiento más completo a través de la comprensión de aspectos fundamentales de fenómenos, de hechos observables o de las relaciones que establecen los entes.

Se recopiló información primaria vía telemática, cuyos datos son confiables, así como válidos, tabulados y sometidos al análisis estadístico. El estudio fue de corte transversal. Las variables consideradas en el estudio, fueron formación holística y la variable habilidades blandas, conocidas como habilidades de las personas, comprenden un conjunto completo sobre el comportamiento social de los individuos, de su intercomunicación y autogestión, las cuales son vitales para el éxito personal y profesional. 
La muestra poblacional, estuvo conformada por 154 estudiantes y 12 docentes del tercer año de bachillerato del plantel, sección vespertina a quienes se le aplicó una encuesta con modalidad de escala de Likert. La variable Formación Holística, contó con las dimensiones: Personal con tres indicadores. Interpersonal con cinco indicadores. Ecológica con cuatro indicadores. Para la variable Habilidades Blandas las dimensiones habilidades Sociales con cuatro indicadores. Empatía con dos indicadores. Autoconciencia con un indicador. Autorregulación con tres indicadores. Motivación con un indicador, a quienes se analizó de manera descriptiva e inferencial.

\section{Tabla 1}

Niveles de interpretación del cuestionario de Formación Holística

\begin{tabular}{|l|c|c|c|}
\hline & Bajo & Medio & Alto \\
\hline Personal & $3-6$ & $7-11$ & $12-15$ \\
Interpersonal & $5-11$ & $12-18$ & $19-25$ \\
Ecológico & $4-8$ & $9-15$ & $16-20$ \\
Formación Holística & $12-27$ & $28-44$ & $45-60$ \\
\hline
\end{tabular}

\section{Tabla 2}

Niveles de interpretación del cuestionario habilidades blandas

\begin{tabular}{|l|c|c|c|}
\hline & Bajo & Medio & Alto \\
\hline Habilidades Sociales & $4-16$ & $17-31$ & $32-44$ \\
Empatía & $2-8$ & $9-15$ & $16-22$ \\
Autoconciencia & $1-3$ & $4-8$ & $9-11$ \\
Autorregulación & $3-12$ & $13-23$ & $24-33$ \\
Motivación & 1 & $4-8$ & $9-11$ \\
Habilidades Blandas & $11-25$ & $26-40$ & $41-55$ \\
\hline
\end{tabular}

Los niveles de interpretación de las variables según sus dimensiones e instrumentos fueron establecidos de manera que los resultados de la información obtenida en los cuestionarios permitan establecer la significancia en la normalidad de su distribución, para lo cual los datos fueron analizados en el sistema estadístico SPSS donde se evidenció la ausencia de normalidad 


\section{Tabla 3}

Formación Holística en la unidad educativa fiscal Vicente Rocafuerte.

\begin{tabular}{|l|c|c|}
\hline & Frecuencia & Porcentaje \% \\
\hline Bajo & 31 & 19 \\
Medio & 63 & 38 \\
Alto & 72 & 43 \\
Total & 166 & 100,00 \\
\hline
\end{tabular}

Fuente: Aplicación de encuesta

El resultado obtenido en la tabla 3, evidencia la existencia de una marcada discrepancia en el criterio de docentes y estudiantes, donde $43 \%$ de los encuestados confirman que en el plantel se desarrolla una enseñanza integral. Luego, el 38\% de encuestados consideran que probablemente si, se aplica el desarrollo de esta enseñanza. Sin embargo, el $31 \%$ niega que este tip0o de formación este implementada en el centro educativo, y tienen la convicción de que no se desarrolla educación holística en el nivel de estudio de bachillerato.

\section{Tabla 4}

Habilidades Blandas en la unidad educativa fiscal Vicente Rocafuerte

\begin{tabular}{lcc}
\hline & Frecuencia & Porcentaje \% \\
\hline Insuficiente & 35 & 21,0 \\
Aceptable & 66 & 40,0 \\
Satisfactoria & 65 & 39,0 \\
Total & 166 & 100,0 \\
\hline
\end{tabular}

Fuente: Aplicación de encuesta

El resultado indica que, el $39 \%$ de encuestados consideran que definitivamente las habilidades blandas son satisfactorias, tanto en docentes como en estudiantes, elevando la confianza y mejorando además la interrelación, el 40\% del personal encuestado, consideran que probablemente esta interrelación debería aplicarse en el centro de estudio, donde es aceptable su nivel de presencia. El 21\% consideró que definitivamente no se enseñan las habilidades blandas mediante la interrelación de docentes y estudiantes, que permita generar un aprendizaje integral, en virtud que es necesario inculcar factores como el respeto, honestidad, compromiso, etc., en los procesos formativos. 


\section{Tabla 5}

Dimensiones de las habilidades blandas en la unidad educativa VR.

\begin{tabular}{|l|c|c|c|c|c|c|}
\hline & $\begin{array}{c}\text { Habilidades } \\
\text { sociales }\end{array}$ & Empatía & $\begin{array}{c}\text { Auto- } \\
\text { conciencia }\end{array}$ & $\begin{array}{c}\text { Auto- } \\
\text { regulación }\end{array}$ & Motivación & $\begin{array}{c}\text { Total } \\
\text { \% }\end{array}$ \\
\hline Insuficiente & 52 & 45 & 23 & 57 & 30 & 25 \\
Aceptable & 95 & 90 & 115 & 92 & 112 & 61 \\
Satisfactorio & 19 & 31 & 28 & 17 & 24 & 14 \\
& 166 & 166 & 166 & 166 & 166 & 100 \\
\hline
\end{tabular}

Fuente: Aplicación de la encuesta

Los resultados del cruce de las dimensiones de las habilidades blandas, evidencian que en los procesos de formación educativa estas tienen un promedio porcentual del $25 \%$ en ser insuficiente, es decir, no hay presencia de las mismas dentro del aula virtual, de igual manera se refleja que un promedio porcentual del $61 \%$ consideran que es aceptable al estar inmerso un fenómeno sanitario pandémico, donde las actuales restricciones han generado una diversidad de situaciones que han afectado el normal desarrollo tanto intelectual como personal de estudiantes y maestros. Luego, un promedio del $14 \%$ establece como satisfactorio al hacer presencia tanto en el salón de clases como en la comunidad educativa del plantel objeto de estudio.

Se desarrolló el análisis inferencial para interpretar la normalidad de la distribución de los datos y la correlación entre las variables. Luego, se utilizó el estadístico de Kolmogórov-Smirnov, en razón de la cantidad de la muestra poblacional procesada que supera los 50 datos, dando una significancia de (Sig.0,000) en cada uno de los elementos considerados.

Tabla 6 Pruebas de normalidad de variable V1 - V2 y sus dimensiones

\begin{tabular}{|lc|c|c|c|c|c|} 
& \multicolumn{3}{c}{ Kolmogórov-Smirnov } & \multicolumn{3}{c}{ Shapiro-Wilk } \\
& Estadístico & gl & Sig. & Estadístico & gl & Sig. \\
\hline Formación Holística &, 203 & 166 &, 000 &, 864 & 166 &, 000 \\
\hline Habilidades Blandas &, 215 & 166 &, 000 &, 868 & 166 &, 000 \\
\hline Dimensión H. Sociales &, 307 & 166 &, 000 &, 831 & 166 &, 000 \\
\hline Dimensión Empatía &, 337 & 166 &, 000 &, 817 & 166 &, 000 \\
\hline Dimensión Autoconciencia &, 353 & 166 &, 000 &, 756 & 166 &, 000 \\
\hline Dimensión Autorregulación &, 292 & 166 &, 000 &, 841 & 166 &, 000 \\
\hline Dimensión Motivación &, 327 & 166 &, 000 &, 809 & 166 &, 000 \\
\hline
\end{tabular}

a. Corrección de significación de Lilliefors

Fuente: Sistema SPSS 
El valor de significancia resultante en la tabla 3 fue menor a $(0,05)$, razón por lo cual indica que no hay normalidad en la distribución de los datos, que exige utilizar el estadístico de correlación de Rho - Spearman.

Tabla 7

Correlaciones no paramétricas V1 y V2.

\begin{tabular}{|c|c|c|c|c|}
\hline & & & $\begin{array}{c}\text { Formación } \\
\text { Holística }\end{array}$ & $\begin{array}{c}\text { Habilidades } \\
\text { Blandas }\end{array}$ \\
\hline \multirow{6}{*}{$\begin{array}{l}\text { Rho de } \\
\text { Spearman }\end{array}$} & \multirow{3}{*}{$\begin{array}{l}\text { Formación } \\
\text { Holística }\end{array}$} & $\begin{array}{l}\text { Coeficiente } \quad \text { de } \\
\text { correlación }\end{array}$ & 1,000 & ,089 \\
\hline & & Sig. (bilateral) & . & 255 \\
\hline & & $\mathrm{N}$ & 166 & 166 \\
\hline & \multirow{3}{*}{$\begin{array}{l}\text { Habilidades } \\
\text { Blandas }\end{array}$} & $\begin{array}{l}\text { Coeficiente } \\
\text { correlación }\end{array}$ & ,089 & 1,000 \\
\hline & & Sig. (bilateral) & ,255 & . \\
\hline & & $\mathrm{N}$ & 166 & 166 \\
\hline
\end{tabular}

Fuente: Sistema SPSS

La tabla 7, establece el tipo de correlación de Rho Spearman entre las variables V1 y V2, dando como resultado, un valor de significancia de $=0,089$ que se interpreta como que las variables formación holística y habilidades blandas tienen una correlación positiva muy baja.

\section{RESULTADOS Y DISCUSIÓN}

La prueba de hipótesis, descarta la hipotesis nula Ho: formación holística no tiene una relación significativa con las habilidades blandas, validando la hipótesis alterna H1: la formación holística tiene una relación significativa con las habilidades blandas, en razón del coeficiente de correlación que es positivo.

El ser humano, manifiesta integridad a base de la comprensión previa de sus actitudes holistas, aportando sus capacidades y tiempo con fines de lograr una formación contínua y trascendente, donde tales cualidades holísticas son procedentes aplicarlas en la actual realidad educativa. Esto debido, a la pérdida de principios y valores que se acentúan cada vez más en el comportamiento juvenil, que luego se verán reflejados en su futura práctica profesional, lo cual, es posible evidenciar en las conflictivas situaciones sociales por las que atraviesa las colectividades, educativas, productivas, comerciales, políticas, económicas, de salud, religiosas y medio ambientales entre otras, donde prevalecen los intereses materiales sobre los humanitarios. El desarrollo de los instrumentos que 
contienen las dimensiones de cada una de sus variables y sus indicadores correspondientes, pueden reflejarse en la tabla 1, donde más del $80 \%$ de los encuestados, manifestaron que mediante una educación holística es posible inducir al estudiante "a aprender ser", esto es, sobreponer las características humanas como persona por encima de lo estrictamente material, siendo colaborativo, cooperativo, respetuoso y ético, en combinación con aquellos conocimientos de la ciencia que hayan sido adquiridos para el buen desempeño en sus actividades socio productivas.

De igual manera refleja un menor porcentaje de encuestados, cuyo criterio es contrario al anteriormente expresado, lo que puede ser interpretado, que es necesario determinar un mínimo nivel de correspondencia entre los actores involucrados en el estudio. En el cuestionario \#16, relacionado con la dimensión ecológica, el resultado de la interrogante, expresa que el $85 \%$ de los encuestados, creen que definitivamente si, se debe orientar a los estudiantes en los procesos formativos, la responsabilidad con la natura y el entorno del cual forma parte, en proteger y cuidar con fines de prevalecer el medio ambiente que es el sustento natural de la vida. El 10\% de los encuestados tienen un criterio contrario al expuesto, razón suficiente en que se analice esta temática con un enfoque educativo.

Es de poner en relevancia, que los cuatro pilares de la educación se fundamentan en aprender ser, el interés en conocer, hacer con responsabilidad y la convivencia armónica con la sociedad, lo que es posible sustentarlo en las dimensiones holística como la ciencia, sociedad, espiritualidad y ecología (Galarreta, 2008, p.57). En el ítem 19, cuya interrogante se vincula con el currículo educativo, el $81 \%$ de personas encuestadas consideran que es necesario una modificación en las planificaciones de los conocimientos y metodologías para la enseñanza y aprendizaje sobre todo en implementar el aspecto humanístico. El 9\% de tiene un diferente criterio, al considerar que el actual sistema formativo en las instituciones, se enfocan en logros inmediatos mediante las competencias y capacidades sobre los conocimientos a ser adquiridos y ofertados en el ámbito laboral. En el cuestionario 18, fundamentado en la dimensión empatía de las habilidades blandas, se preguntó sobre fomentar la integridad para generar conciencia política en los estudiantes, el $65 \%$ consideran que definitivamente se requiere educar sobre aquello al estudiante, el $20 \%$ consideran que debe dársele un enfoque diferente.

La interrogante 17, está orientada a obtener una respuesta, si las habilidades blandas son innatas o aprendidas, se obtuvo que un $86 \%$, contestaron que definitivamente si, estas 
habilidades son generadas en los procesos formativos de la educación. En contraposición, un $9 \%$ de los elementos encuestados piensan que son habilidades heredadas. Estudios de Bryan y Harter (1980), indican "una lista de habilidades blandas necesarias para lograr una formación educativa exitosa y profesional se describe en el aprendizaje basado en proyectos que implica ser comunicativo, empático, colaborador, con iniciativa y ética en la función del trabajo asignado" (p.24). De igual manera, Ortega (2017), consideró que, "los niños y jóvenes de hoy no son los mismos de ayer, estos adquieren conocimientos por diversos medios y formas, razón suficiente para inculcar normas de comportamiento social con habilidades blandas".

Los instrumentos para desarrollar las encuestas, fueron validados por cinco expertos, las que estaban constituidas por 10 interrogantes que vinculaban a la variable formación holística y a la variable habilidades blandas las cuales fueron calificadas como con pertinencia, relevancia y claridad, que permitieron desarrollar una prueba piloto con resultados del alfa de Cronbach $=0,826909379$ lo que certificó la fiabilidad de la aplicación de los instrumentos. Una vez confirmada la confiabilidad de los ítems, se desarrolló la encuesta a la muestra poblacional previamente establecida donde se buscó identificar una relación significativa entre las variables, mediante el análisis de normalidad que para el presente caso donde los elementos de las variables sobre pasan en un número mayor a 50 datos. La prueba de normalidad según Kolmogórov-Smirnov, resultó con una significancia $=0,000$, que indica ausencia de normalidad y que se orientó para aplicar una correlación no paramétrica mediante Rho Spearman, cuyo indicador significante entre las variables formación holística y habilidades blandas fue $=0,748$, que se interpreta como una correlación "positiva alta" según la escala de valores del coeficiente de correlación.

Estos resultados validan la hipótesis nula que se planteó inicialmente. A demás se obtuvieron otras cifras sobre el aspecto descriptivo, que a continuación se exponen:

El resumen del procesamiento del caso entre las variables V1 y V2, cuenta con grado de libertad (gl) de 166 unidades, donde fueron analizados en un 100\%. La descripción de los datos de la variable formación holística, indica un 95\% de intervalo de confianza, con una media de 1,6627 y desviación de error de 0,08041, una media de 1,00, varianza con valores de 1,073, desviación de la varianza 1,03604, la asimetría 1,708, desviación de la asimetría 0,188. Curtosis con un indicador de 2,240 y desviación del 0,375. 
En la variable habilidades blandas, se tienen los siguientes datos descriptivos: la media es de 1,6807 con desviación de error por 0,8504 con el 95\% de intervalo de confianza. La mediana 1,00, de igual manera la varianza con indicador del 1,200. La asimetría 1,754 con desviación de error por 0,188 . Curtosis 2,320 con desviación de 0,375. Es de considerar que el muestreo utilizado para desarrollar las pruebas de normalidad y de correlación es aleatorio, en virtud que cada uno de los elementos de la población tienen características similares. Esta prueba de normalidad de Kolmogorov, es utilizada cuando los elementos a ser analizados superan los 50, y su función es comparar la función de distribución acumulada empírica de los datos de la muestra con la distribución esperada cuando los datos son normales. De igual manera, la prueba de correlación Rho- Spearman, se aplica cuando no existe normalidad en la distribución, por tal razón, se la utiliza para determinar la correlación existente entre las variables analizadas, de acuerdo al valor de su significancia, puede ser nula, baja o alta.

Haciendo una recopilación de la información obtenida, se considera que la actual formación holística en los estudiantes de la unidad educativa fiscal Vicente Rocafuerte, evidencian que, del total de los 15 participantes escogidos de la muestra para el desarrollo de la prueba piloto, en el ítem 14, existe una diversidad de criterios, donde el $20 \%$ de los encuestados afirman que la educación holística es aplicada en la enseñanza de los estudiantes, el $60 \%$ mantiene el criterio de probablemente si es desarrollada esta variable, sin poder afirmarlo con seguridad. El 7\% no tiene una decisión definida y el 13\% lo niega categóricamente. Los resultados porcentuales obtenidos en el resto de ítems, sobre formación holística y sus dimensiones, personal, interpersonal y ecológica, verifican la existencia de un vacío de la presencia de este proceso educativo en el nivel de bachillerato de plantel objeto de estudio.

La formación holística de la teoría de Jan Smuts (1926), y habilidades blandas de Goleman D. (1999), evidencia una relación o incidencia, que permite plantear una propuesta para mejorar la formación educativa del estudiante del plantel. Los resultados obtenidos en el ítem 19, relacionado con la variable 2, se destaca que el $67 \%$ de los encuestados, confirman la necesidad de modificar el currículo educativo para el bachillerato actualmente vigente, con el fin potenciar el desarrollo de las habilidades blandas, el 13\%, manifestó la probabilidad para que ello se dé. 
En los demás ítems, vinculados con la variable 2 y sus dimensiones, los resultados obtenidos reflejan la ausencia de habilidades blandas como producto de la formación educativa en el nivel de estudio investigado, lo cual es un importante justificativo para desarrollar un programa que permita su producción y desarrollo.

La validación de los instrumentos, permitieron direccionar la investigación, donde se evidenció un diagnóstico mediante el cual es posible proponer un programa educativo para dar una solución definitiva a la problemática identificada sobre la relación entre formación holística y las habilidades blandas de los estudiantes.

Es de considerar que, el principio general del holismo fue mentalizada por Aristóteles de manera precisa en sus escritos metafísicos, proyectando una visión más allá de la física, interpretada como que, "el todo es mayor que la suma de sus partes". Esto es, un vehículo es más que la suma de sus neumáticos, motor y carrocería. Es decir, por separado, las partes nunca podrían realizar un viaje. Además, hay que saber conducir un vehículo. Tomando en consideración este aspecto, se lo puede simular como el estudio de una temática o el planteamiento de un problema donde se deben considerar todos sus elementos o componentes, incluyendo los factores intangibles existentes.

Así, la perspectiva holista, refiere en que, los sistemas funcionan como conjuntos y, por lo tanto, este funcionamiento no es comprendido, si solo se tienen en cuenta las partes que conforman el todo. Por lo tanto, la orientación del holismo se fundamenta en cómo estudiar una estructura como un todo y en un contexto específico a tratarse. "La holística se refiere a la manera de ver las cosas enteras, en su totalidad, en su conjunto, en su complejidad, pues de esta forma se pueden apreciar interacciones, particularidades y procesos que por lo regular no logran percibirse al estudiarse por separado”. (Briceño et.al, 2010,p.74).

Los paradigmas holísticos son: "El sistema es mayor que la suma de sus partes. Las partes no se pueden comprender sin su relación con el sistema en su conjunto". "El conocimiento en todas sus facetas y la esencia de lo emocional, lo intuitivo y lo espiritual se complementan y se potencian". "Las soluciones y los efectos repercuten sobre el sistema tanto como los problemas y las causas. Desde este punto de vista, un efecto puede llegar a convertirse en una causa en sí”. "El conocimiento es indivisible en disciplinas diferentes y en modos de experiencias". "Los seres humanos nos encontramos inmersos en los sistemas naturales y la vida humana depende de la naturaleza para su supervivencia". "La 
adquisición gradual de capacidades permite resolver nuestros propios problemas y transformar nuestras vidas. Esto permite disminuir la dependencia de expertos y especialistas". "El observador y lo observado se encuentran en relación recíproca". "Los fenómenos y sucesos son contemplados desde una perspectiva dinámica e interconectada tanto en el tiempo como en el espacio”. (Briceño, et.al, 2010).

Por otro lado, las habilidades blandas son el resultado de una combinación de habilidades sociales, de comunicación, de forma de ser, de acercamiento a los demás, entre otras cosas; que forjan a una persona ser capaz de relacionarse y comunicarse de manera efectiva y eficiente con otros semejantes, donde tal aspecto es muy apreciado y demandado por los actuales sistemas empresariales (Gestión, 2020). La reflexxión de que una adecuada inversión en habilidades blandas tales como el liderazgo, cooperativismo, inteligencia emocional, creatividad, responsabilidad, creatividad, así como, el pensamiento crítico, reduciría de manera significativa el volumen de puestos de trabajos perdidos en la automatización hasta en un $10 \%$.

Las habilidades blandas vinculadas al sector laboral, comercial y productivo que son actualmente demandadas, son: la gestión de conflictos, gestión del tiempo, manejo del estrés, habilidades de comunicación, cultura de empresa, servicio al cliente, inteligencia emocional, productividad personal, narración y gestión del cambio. Las organizaciones empresariales a nivel globalizado, han direccionado sus demandas en profesionales que han sido capaces de desarrollar sus habilidades blandas en paralelo a sus estudios. Aquí la importancia de continuar con la formación, aun cuando ya se tenga un título académico, en virtud que, la oficina de recursos humanos, han dejado de dar tanta relevancia al número de títulos o diplomas con los que cuenta un determinado candidato para un puesto de trabajo, el mismo que su evaluación se enfoca el desarrollo y capacidades de sus habilidades blandas o transversales. Por lo tanto, a más de los conocimientos científicos curriculares adquiridos en los centros formativos educativos, conocidos como habilidades duras, la evaluación de las habilidades blandas se centra en el desarrollo práctico sobre las aptitudes, personalidad, criterio y responsabilidad. Entre las habilidades blandas más relevantes se tiene: las habilidades comunicativas, la creatividad, responsabilidad, honestidad, proactividad, resiliencia, trabajo en equipo, empatía, adaptación al cambio y orientación al cliente. En el ámbito académico, cada vez hay mayor consciencia en la importancia de formar a la población estudiantil con 
habilidades transversales, con el objetivo de que no solo se dediquen en absorber conocimiento empírico, sino que se complemente sus capacidades con una formación humanística. Luego, la tecnología y sus constantes adelantos, requieren que las nuevas generaciones desarrollen habilidades blandas desde su temprana edad, situación que aun no ha sido considerada en los currículos académicos educativos, en virtud que estas serán el principal aspecto calificado para lograr un empleo.

\section{CONCLUSIÓNES}

El análisis de correlación entre las variables formación holística y habilidades blandas en la unidad educativa fiscal Vicente Rocafuerte - Guayaquil, 2020; es positiva muy baja, según el coeficiente de correlación que refleja un valor de 0,089. Sin embargo, no es suficiente para el desarrollo de habilidades blandas y sus dimensiones para una eficiente formación humanística y académica en la población estudiantil.

Un programa de formación holística no se evidencia en el desarrollo curricular de la institución. El currículo educativo ecuatoriano, con contenidos sobre principios y valores humanísticos del holismo no están desarrollados de manera adecuada y eficiente, con fines de generar las habilidades blandas en los procesos formativos de la población estudiantil del plantel.

El nivel de habilidades blandas en el plantel es satisfactorio para el 39\% de encuestados, así como aceptable para el $40 \%$ e insuficiente para el $21 \%$, donde este último indicador establece que de cada 100 estudiantes que se forman en el plantel, 21 no asumen una pertenencia social, empatía, autoconciencia, autorregulación o motivación, ligada a valores, principios, respeto y responsabilidad con los semejantes.

El nivel de correlación entre las variables formación holística (V1) y la variable Habilidades blandas (V2), es positiva muy baja, al reflejar un coeficiente de 0,089 según la prueba no para métrica de Rho Spearman

En la actualidad en la institución educativa objeto de estudio, no existe un programa de formación holística para el tercer año de bachillerato, que pueda ser aplicado en los procesos formativos del plantel para optimizar las habilidades blandas en la población estudiantil. Sin embargo, según el desarrollo del análisis descriptivo de las cinco dimensiones de la variable habilidades blandas (V2), estas reflejan características con un nivel aceptable. 


\section{REFERENCIAS BIBLIOGRÁFICAS}

Álvarez Cáceres , R. (2017). Estadística aplicada a las ciencias de la salud. Madrid: Diaz de Santos.

Andrighetti, P. (24 de octubre de 2013). Habilidades blandas, capacitando para la vida.

Obtenido de Hacer familia: https://hacerfamilia.cl/2012/10/habilidades-blandascapacitando-para-la-vida/

Briceño, J., Cañizares , B., Lobo, H., Moreno, E., Velásquez , I., \& Rivas, Y. (2010). la holística y su articulación con la generación de teorias. Educere, 12.

Cardenas, A., \& Jiménez Hernández, N. (2017). Acceso universal a la información. Scielo.

Carvajal Monterrosa, A. B. (21 de Junio de 2019). Sociedad de información y conocimiento. Obtenido de formación ib: http://formacionib.org/noticias/?Sociedad-de-informacion-y-conocimiento

Carvajal, L., Ormeño, J., \& Sanz, L. (2019). Atención al cliente. Madrid: FBP.

Cauas, D. (2015). Definición de las variables, enfoque y tipo de investigación. Bogotá: UEB.

Corbetta, P. (2015). Metodología y Técnica de Investoigación Social. Madrid: Mc GrawHill.

De la Cruz Sandoval, V. V. (2020). Habilidades blandas en las relaciones interpersonales en docentes de la Institución educativa Karol Wojtyla. Lima: $\mathrm{UCV}$.

Espino de Lara, R. (s.f.). Educación Holística. Revista Iberoamericana de Educación , 9. Espinoza Mina, M. (2020). Habilidades blandas en la educación y la empresa . Uisrael, 56.

Galarreta Ugarte, M. (2018). Metodología de Educación Holística y el desarrollo de competencias comunicativas. Lima: UTP.

García Avendaño, P. (2016). Introducción a la Investigación Bioantropológica. Caracas: $\mathrm{UCV}$.

Gestión. (26 de junio de 2020). Habilidades blandas demandadas por el mercado. Obtenido de https://gestion.pe/economia/management-empleo/10-habilidadesblandas-demandara- 
Gluyas Fitch, R. I., Esparza Parga, R., Romero Sánchez, M., \& Rubio Barrios, J. E. (2015). Modelo de educación holística: propuesta para la formación del ser humano. Actualidades Investigativas en Educación, 25.

Goleman, D. (2001). Inteligencia emocional.

Llamazares, A. M. (2017). Epistemología holística. CONICET, 58.

López Arrillaga, C. (2018). Educación holistica desde una perspectiva humanística. Caracas: ULAC.

Marrero Sánchez , O. (2018). Habilidades blandas necesarias para la formación integral del estudiante universitario. Ecociencia, 18.

Marrero Sánchez, O., Mohamed Amar, R., \& Xifra Triadú, J. (2018). Habilidades Blandas: necesarias para la formación del estudiante universitario. Ecociencia.

Megías Tortosa , A., \& Lozano Serrano, L. (2019). Desarrollo socio afectivo. Madrid: Editex.

Ministerio de Educación del Ecuador. (2016). perfil del bachillerato ecuatoriano: desde la educación hacia la sociedad. Quito: MEE.

Niño Rojas, V. (2013). Metodología de la investigación. Bogotá: EU.

Ortega Santos, C. E. (2017). Desarrollo de habilidades blandas desde edades tempranas. Guayaquil: Ecotec

Rodriguez Gómez , D., \& Valldeoriola Roquet, J. (2014). Metodología de la investigación. Cataluña: UOC.

Rodriguez Marcos , A. (2020). El prácticum factyor de calidad en la formación del profesorado de secundaria. Barcelona: Octaedro.

Rodriguez Torres, D. A. (2019). Influencia de las humanidades en la construcción holística de la ciudadanía. Bucaramanga: UST.

Romero, D. (7 de Marzo de 2020). Rockcontent.com. Obtenido de Ambiente Virtuales de Aprendizaje: $\quad$ https://rockcontent.com/es/blog/ambientes-virtuales-deaprendizaje/

Semplades. (2013). Plan nacional del buen vivir. Quito: MEE.

Smuts, j. (1926). Holismo. Sud africa.

Tito Maya, M., \& Serrano Orellana, B. (2016). Desarrollo de soft skills una alternativa a la escasez de talento humano. INNOVA, 76. 
Tolentino Quiñones, H. (2020). Habilidades sociales y estrategias didácticas para la formación del liderazgo desde la educación básica. Educación, 20.

UNESCO. (2018). Tres dimensiones de requerimientos a la formación inicial docente. competencias S.21, Inclusión, Ciudadanía (pág. 19). Cochabamba: Educación 2030.

Vallejos Carrasco, A. (2019). Habilidades blandas y su influencia en el desempeño en el personal post venta. Chiclayo: UCSTB. 